Ebisu Ebisu

Études japonaises Études japonaises

$58 \mid 2021$

2011-2021 : crises, ruptures et nouvelles dynamiques.

Dix ans après la triple catastrophe du 11 mars

\title{
La vie des réfugiés volontaires de l'accident nucléaire
}

原発区域外避難者はどう生きてきたか

The Life of the Volunteer Evacuees of the Fukushima Nuclear Accident

\section{Shōichirō Takezawa}

Traducteur : Anne Gonon

\section{CpenEdition}

\section{Journals}

Édition électronique

URL : https://journals.openedition.org/ebisu/6037

DOI : $10.4000 /$ ebisu.6037

ISSN : 2189-1893

Éditeur

Institut français de recherche sur le Japon à la Maison franco-japonaise (UMIFRE 19 MEAE-CNRS)

Édition imprimée

Date de publication : 1 novembre 2021

Pagination : $249-270$

ISSN : 1340-3656

\section{Référence électronique}

Shōichirō Takezawa, "La vie des réfugiés volontaires de l'accident nucléaire », Ebisu [En ligne], 58 |

2021, mis en ligne le 01 novembre 2021, consulté le 13 avril 2022. URL : http://

journals.openedition.org/ebisu/6037 ; DOI : https://doi.org/10.4000/ebisu.6037 


\section{La vie des réfugiés volontaires de l'accident nucléaire}

TAKEZAWA Shōichirō

原発区域外避難者はどう生きてきたか

竹澤尚一郎

The Life of the Volunteer Evacuees of the Fukushima Nuclear Accident

TAKEZAWA Shōichirō

Mots-clés : accident nucléaire, réfugiés, conditions de vie, enquête sociale, indemnisations, procès

L'auteur : Takezawa Shōichirō est professeur émérite au musée national d'Ethnologie et au Collège doctoral de recherche avancée et chercheur invité à la Fondation France-Japon (EHESS). Il est spécialiste d'anthropologie sociale et d'histoire africaine. Après un doctorat à l'EHESS, il a été maître de conférences à l'université du Kyūshū.

Résumé : Laccident de la centrale nucléaire de Fukushima Daiichi a fait
170000 évacués, et leur nombre dépasse encore les 40000 dix ans plus tard. Le gouvernement japonais a fixé des normes d'indemnisation pour les évacués et a ordonné à Tepco de les payer. Beaucoup d'entre eux sont néanmoins mécontents du montant de l'indemnisation et du manque d'excuse du gouvernement: environ 15000 personnes ont déposé 28 poursuites contre Tepco et le gouvernement. Sur la base des déclarations soumises au tribunal et de nos questionnaires, cet article s'efforce de dresser un document de vie de 171 personnes évacuées (54 ménages) qui ont déposé une plainte devant le tribunal de Kyoto. 
ンキーワード

原発事故、難民、生活環境、社会調査、賠償、 民事訴訟

\section{著者}

竹澤尚一郎 : 国立民族学博物館・総合研究大 学院大学名誉教授。フランス社会科学高等研 究院日仏財団招聘研究員。社会人類学、アフ リカ史専攻。フランス社会科学高等研究院博 士課程修了 (民族学博士)。日本学術振興会特 別研究員を経て、九州大学文学部助教授、教授。
要旨

福島第一原子力発電所の重大事故は、最大で 約 17 万人の避難者を生み、1 0 年後の時点 でも避難者は 4 万人を超えている。日本政府 は避難者に対する賠償基準を定めて東京電力 に支払いを命じたが、多くの避難者は低額の 賠償額と政府の謝罪のないことを不服とし、 東京電力と国に対して約 15,000 人が 28 件の 訴訟を起こしている。本稿は、京都地方裁判 所に提訴した避難者 54 世帯、171 名が裁判 所に提出した陳述書と私たちが実施したアン ケートをもとに、彼らの避難生活の実態を明 らかにしょうとするものである。
Veywords: Nuclear Accident, Refugees, Living Conditions, Social Survey, Compensation, Lawsuit

The Author: Takezawa Shōichirō is a professor emeritus at National Museum of Ethnology and Graduate School for Advanced Studies, and visiting researcher at France-Japan Foundation of EHESS. He is specialised in Social Anthropology and African History. After a PhD at EHESS, he became associate professor at Kyūshū University.

Abstract: The accident at the Fukushima Daiichi Nuclear Power Station resulted in 170,000 evacuees, and 10 years on their number still exceeds 40,000. The Japanese government set the standards of compensation that it ordered TEPCO to pay evacuees. However, many evacuees are dissatisfied with the compensation amount and by the absence of a government apology, with some 15,000 people filing 28 lawsuits against TEPCO and the government. Based on the statements submitted to the court and on our questionnaires, this paper seeks to establish a "life document" of 171 evacuees from 54 households who filed a complaint with the Kyoto District Court. 


\section{La vie des réfugiés volontaires de l'accident nucléaire}

TAKEZAWA Shōichirō

原発区域外避難者はどう生きてきたか

竹澤尚一郎

The Life of the Volunteer Evacuees of the Fukushima Nuclear Accident

TAKEZAWA Shōichirō

Mots-clés : accident nucléaire, réfugiés, conditions de vie, enquête sociale, indemnisations, procès

L'auteur : Takezawa Shōichirō est professeur émérite au musée national d'Ethnologie et au Collège doctoral de recherche avancée et chercheur invité à la Fondation France-Japon (EHESS). Il est spécialiste d'anthropologie sociale et d'histoire africaine. Après un doctorat à l'EHESS, il a été maître de conférences à l'université du Kyūshū.

Résumé : Laccident de la centrale nucléaire de Fukushima Daiichi a fait
170000 évacués, et leur nombre dépasse encore les 40000 dix ans plus tard. Le gouvernement japonais a fixé des normes d'indemnisation pour les évacués et a ordonné à Tepco de les payer. Beaucoup d'entre eux sont néanmoins mécontents du montant de l'indemnisation et du manque d'excuse du gouvernement: environ 15000 personnes ont déposé 28 poursuites contre Tepco et le gouvernement. Sur la base des déclarations soumises au tribunal et de nos questionnaires, cet article s'efforce de dresser un document de vie de 171 personnes évacuées (54 ménages) qui ont déposé une plainte devant le tribunal de Kyoto. 
】キーワード

原発事故、難民、生活環境、社会調査、賠償、 民事訴訟

\section{著者}

竹澤尚一郎 : 国立民族学博物館・総合研究大 学院大学名誉教授。フランス社会科学高等研 究院日仏財団招聘研究員。社会人類学、アフ リカ史専攻。フランス社会科学高等研究院博 士課程修了 (民族学博士)。日本学術振興会特 別研究員を経て、九州大学文学部助教授、教授。
要旨

福島第一原子力発電所の重大事故は、最大で 約 17 万人の避難者を生み、1 0 年後の時点 でも避難者は 4 万人を超えている。日本政府 は避難者に対する賠償基準を定めて東京電力 に支払いを命じたが、多くの避難者は低額の 賠償額と政府の謝罪のないことを不服とし、 東京電力と国に対して約 15,000 人が 28 件の 訴訟を起こしている。本稿は、京都地方裁判 所に提訴した避難者 54 世帯、171 名が裁判 所に提出した陳述書と私たちが実施したアン ケートをもとに、彼らの避難生活の実態を明 らかにしょうとするものである。

\section{Yeywords: ?}

The Author: Takezawa Shōichirō is a professor emeritus at National Museum of Ethnology and Graduate School for Advanced Studies, and visiting researcher at France-Japan Foundation of EHESS. He is specialised in Social Anthropology and African History. After a PhD at EHESS, he became associate professor at Kyūshū University.

Abstract: The accident at the Fukushima Daiichi Nuclear Power Station resulted in 170,000 evacuees, and 10 years on their number still exceeds 40,000. The Japanese government set the standards of compensation that it ordered TEPCO to pay evacuees. However, many evacuees are dissatisfied with the compensation amount and by the absence of a government apology, with some 15,000 people filing 28 lawsuits against TEPCO and the government. Based on the statements submitted to the court and on our questionnaires, this paper seeks to establish a "life document" of 171 evacuees from 54 households who filed a complaint with the Kyoto District Court. 


\section{La vie des réfugiés volontaires de l'accident nucléaire}

TAKEZAWA Shōichirō*

\section{Introduction}

Le 11 mars 2011 s'est produit le grand séisme du Japon de l'Est, dont l'épicentre se situait au large du département de Miyagi. Dans la centrale nucléaire de Fukushima Daiichi, de la société d'électricité de Tokyo (Tepco), l'alimentation en électricité nécessaire au refroidissement a été coupée en raison du séisme et du tsunami, rendant impossible le contrôle de l'enceinte de confinement du réacteur et entraînant la fusion de son cœur. À 19 h 30, le jour même, le gouvernement a déclaré « l'état d'urgence nucléaire ", demandant aux habitants vivant dans un périmètre de $3 \mathrm{~km}$ autour de la centrale d'évacuer, et à ceux qui vivaient dans un périmètre de $30 \mathrm{~km}$ de se cloîtrer chez eux. Le lendemain, le 12 mars, le périmètre d'évacuation a été élargi à $20 \mathrm{~km}$, à cause de l'explosion du réacteur $\mathrm{n}^{\circ} 1$. Par la suite, les réacteurs 2 et 3 sont tour à tour entrés en fusion et ont explosé, générant des rejets radioactifs de forte puissance qui se sont dispersés audelà du périmètre des $20 \mathrm{~km}$ de la "zone de vigilance ». C'est-à-dire que la dissémination de grandes quantités de particules du fait de l'explosion du réacteur $\mathrm{n}^{\circ} 3$ le 14 mars, puis du n ${ }^{\circ} 2$ le 15 mars, suivie le même jour d'une explosion dans le bâtiment du réacteur $\mathrm{n}^{\circ} 4$, a contraint le gouvernement à demander également aux habitants des communes situées au-delà des

* Professeur émérite au musée national d’Ethnologie et au Collège doctoral de recherche avancée 
$20 \mathrm{~km}$ dans les zones où la radioactivité était élevée telles Iitate et Katsurao, d'évacuer leurs lieux d'habitation pour se rendre dans la « zone de préparation à l'évacuation ». Il a désigné également une "zone prête à l'évacuation en cas d'urgence", et demandé aux habitants dans le périmètre de 20 à $30 \mathrm{~km}$ soit de s'y rendre soit de rester confinés chez eux ${ }^{1}$. Cela concernait 150000 habitants que nous nommons, dans ce texte, les « réfugiés de la zone d'évacuation ».

Mais la situation a empiré. Entre le 14 et le 16 mars, période où la quantité de radioactivité était forte, le vent dissémina les particules radioactives suspendues dans l'air, vers la partie centrale du département de Fukushima où se trouvent des villes fortement peuplées telles que les villes de Fukushima, de Kōriyama et Iwaki ainsi que vers les départements de Ibaraki, et de Tochigi ${ }^{2}$. Dans les villes de Fukushima et de Kōriyama, on enregistra des quantités de radioactivité de l'ordre de 20-30 microsieverts par heure, soit 1000 fois plus qu'en temps ordinaire et beaucoup d'habitants pris de panique décidèrent de fuir. Un plaignant au procès de Kyoto, qui habitait à Kōriyama évoque la situation d'alors : "Dans la société où je travaillais, après l'accident, on a donné aux employés des vêtements de protection, des lunettes protectrices et des masques, et le chef nous a dit «envisagez la possibilité de vous réfugier hors du département. " Une enquête de grande ampleur menée auprès de toutes les mères ayant un enfant de trois ans qui vivent dans les municipalités situées dans la zone centrale du département de Fukushima, montre que seules 29,1\% d'entre elles déclarent ne jamais avoir eu besoin d'évacuer alors que la grande majorité des foyers

1. Département de Fukushima, "À propos de l'évolution des zones de refuge ". https://www.pref.fukushima.lq.jp/site/portal/cat01-more.html (dernière consultation le 3 juin 2019).

2. Dû à un vent d'ouest qui souffla après l'accident nucléaire, une grande partie de la radioactivité se dispersa au-dessus de l'océan pacifique. Au large de Fukushima, le porteavion à propulsion nucléaire Ronald Reagan mena des activités d'aide humanitaire sous le nom d'opération Tomodachi au cours desquelles de nombreux marins furent irradiés. Ils intentèrent un procès au gouvernement américain mais ne purent recevoir d'indeminités, cette intervention ayant été considérée comme faisant partie de l'activité militaire (Tainaka \& Tsujimoto 2018). 
ayant des enfants en bas âge ont évacué tous au moins une fois (Matsutani et al. $2014: 72)^{3}$.

Les municipalités de cette zone centrale sont placées dans le périmètre des zones refuges déterminées par le gouvernement, et si on y ajoute la ville d'Iwaki située au sud de la centrale nucléaire de Fukushima, cela constitue une population de 1435000 habitants et forment, littéralement, le cour du département de Fukushima. La quantité de radioactivité de cette zone dépassait largement les $5 \mathrm{mSv} / \mathrm{an}$, correspondant au seuil d'évacuation forcée après la catastrophe de Tchernobyl, mais l'État et le département de Fukushima, craignant l'apparition d'un grand nombre de réfugiés et le risque d'un chaos économique et social, ont relevé le seuil pour l'évacuation à $20 \mathrm{mSv} / \mathrm{an}$. Cette décision s'appuyait sur la déclaration du 21 mars 2011 de la Commission internationale de la protection radiologique, mais elle correspondait au cadre d'une situation d'urgence. La commission réclamait pour le long terme un seuil de $1 \mathrm{mSv} / \mathrm{h}$, seuil de sécurité que le gouvernement japonais, lui aussi, avait fixé avant l'accident. (Satō \& Taguchi 2016 : 96). Résultat de l'adoption contrainte d'un dispositif aussi inhumain, les habitants de la zone centrale du département de Fukushima et ceux des départements limitrophes, tels Tochigi et Ibaraki craignant la radioactivité se sont réfugiés dans d'autres départements plus lointains. Ils sont considérés comme des "réfugiés volontaires " (ou "réfugiés hors zone d'évacuation ") De ce fait, ils ne reçurent qu'une aide minimale du gouvernement et de faibles dédommagements de la part de Tepco. Ainsi, bon nombre de réfugiés hors zone durent faire face à des difficultés multiples.

Parmi l'ensemble des réfugiés du département de Fukushima, on distinguait, en 2011, 150000 personnes vivant dans les zones refuges et 60000 personnes vivant hors de ces zones, et huit ans après l'accident nucléaire, à la fin 2019, le nombre de réfugiés hors du département de Fukushima dépassait les 30000 personnes. Hormis les publications qui ont suivi immédiatement la catastrophe du Japon de l'Est, il n'existe qu'un nombre limité d'ouvrages abordant la façon de vivre de ces personnes, à savoir les difficultés vécues par les réfugiés hors zone et la façon dont les aides gouvernementales et les indemnisations ont été perçues - dans quel

3. Selon cette enquête, $30,5 \%$ des personnes ont une expérience d' "évacuation de moyen et long termes ", 40,4\% ont vécu une "évacuation temporaire ». (ibid. : 72) 
sens peut-on dire qu'elles sont insuffisantes? À l'exception de quelques recherches (Yamane 2013; Yoshida 2016; Takehashi \& Nakatani 2018, 2019; Tsujiuchi \& Masuda 2019), les travaux manquent. Le présent article vise à compléter ces recherches. Nous allons décrire les conditions de vie des réfugiés en nous appuyant sur les mémoires judiciaires préparés pour le procès de Kyoto qui s'est tenu au tribunal de Kyoto et qui opposait ces réfugiés à l'État et à Tepco, et sur l'enquête conduite à partir de ces documents.

\section{Conditions de l'étude}

Nous allons en premier lieu expliquer le processus qui a mené à la réalisation de ce présent travail. Tout a débuté par une demande émanant du groupe des avocats de Kyoto de travailler à "rendre visibles " les conditions de vie réelles et les difficultés des réfugiés qui étaient aussi plaignants. Les plaignants étaient composés de 56 foyers et 171 personnes (au départ 58 foyers), et avaient rédigé un mémoire avec l'aide de ce groupe d'avocats pour plaider au tribunal les conditions de préjudice. Ce mémoire comprenait 30 pages et détaillait les rubriques telles que le lieu de vie que l'État avait fixé, l'âge, la composition des foyers, les professions, l'expérience d'évacuation, les conditions de vie après être devenus réfugiés. Cette grande variété de données ne permettait pas de comprendre les tendances générales concernant les réclamations et le sens donné à ce procès par chaque plaignant; on nous a donc demandé de poursuivre la tâche afin de saisir ces tendances.

Un précédent à un tel travail existe; ce sont les avis juridiques présentés au tribunal régional de Niigata qu'avait reçus notamment la professeure associée Takahashi Wakana, à l'université Utsunomiya, et les articles qui en sont issus (Takahashi \& Koike 2018, 2019). L’analyse qui en a été faite s'appuie sur les mémoires des 804 plaignants (soit 237 foyers) et doit être considérée comme un modèle tant par l'importance de la population concernée et l'exhaustivité des rubriques que par la précision et le détail de l'analyse. Par ailleurs, plusieurs enquêtes sur les réfugiés avaient déjà été menées par le département de Niigata, dont les 72 rubriques du questionnaire avaient servi à élaborer ces mémoires; ainsi le contenu des mémoires et le classement des notes étaient clairement établis. En revanche, dans le procès de Kyoto, aucun système de rubriques n'avait été préalablement élaboré pour 
fabriquer les mémoires, ce qui en fait des documents différents de ceux du procès de Niigata.

Nous avons suivi la procédure suivante pour analyser les mémoires du procès de Kyoto. D’abord, nous avons élaboré un questionnaire composé de 60 rubriques - à partir des 72 rubriques qui constituaient la base du document utilisé lors du procès de Niigata, nous en avons supprimé 12 qui ne sont presque pas répertoriées dans les mémoires de Kyoto. Ensuite, nous avons relu ces dernières en suivant le questionnaire, et dans le cas où il y avait une remarque concernant une question, nous avons marqué la réponse correspondante, et dans le cas où il n'y en avait pas, nous n'avons rien annoté. Ensuite nous avons fait passer aux plaignants le questionnaire, leur demandant de vérifier chaque rubrique annotée et de compléter les parties laissées vides. Nous avons récupéré les enquêtes renvoyées aux avocats et analysé les résultats, sur lesquels s'appuie la partie principale de cet article.

Sur 56 foyers à qui avait été adressé le questionnaire, 56 ont répondu, donc un retour de $100 \%$, un taux exceptionnel pour une enquête ${ }^{4}$. Cela témoigne du fort intérêt que chaque plaignant portait au procès, de la passion du groupe des avocats, et des relations de confiance entre plaignants et avocats. La crainte de subjectivité dans la rédaction des mémoires dont les avocats auraient pu faire preuve, nous a conduit à relire entièrement ces documents, à marquer d'une croix les réponses au questionnaire, rectifiées ou laissées vides en se rappelant le travail de rédaction des plaignants, et à l'issue de ce travail nous estimons que la volonté des plaignants a été autant que possible respectée. En outre, dans les questionnaires qu'ils ont retournés, les plaignants ont ajouté des témoignages très vivants, et en les insérant dans le présent article, nous pensons pouvoir transmettre fidèlement les conditions de vie des plaignants, et permettre une analyse quantitative et qualitative des notes.

Le présent article présente un tableau des données recueillies dans les 56 mémoires et rend compte des caractéristiques des plaignants du procès au Kyoto ${ }^{5}$.

4. Dans les enquêtes en sociologie, généralement, le taux de retour est de l'ordre de $20 \%$ lors d'enquête envoyée par la poste (Seiyama 2004 : 68).

5. Avec l'accord de l'auteur, nous avons réduit l'article à la partie concernant les plaignants du procès de Kyoto. La première version de l'article incluait de nombreuses 


\section{Tableau des caractéristiques des plaignants apparaissant dans les mémoires du procès}

\section{1. Caractéristiques sociales des foyers de plaignants}

Nous allons tout particulièrement nous intéresser aux rubriques qui présentent les caractéristiques des plaignants. En outre, nous prêterons attention à ce qui les différencie de ceux du procès de Niigata, en nous aidant de la recherche de Takahashi \& Koike $(2018,2019)$.

Pour ce faire, nous avons retenu trois dimensions : le lieu de vie, l'âge et la profession.

- Lieu de vie après l'accident nucléaire : en dehors d'un réfugié venant d'une zone de retour difficile, et d'un autre réfugié venant d'une zone de préparation au refuge d'urgence, tous les plaignants sont des réfugiés dits " refugiés hors zone d'évacuation ". Beaucoup - 2 de Miyagi, 2 d'Ibaraki, 2 de Chiba - ne viennent pas du département de Fukushima, mais de zones où le niveau de radioactivité était très élevé; en outre, un foyer vient du département de Fukushima, mais d'une zone exclue de la loi d'aide aux victimes du désastre. Ils n'ont pas droit aux indemnisations versées par Tepco et c'est une des raisons du procès.

- Âge des plaignants : 71,5\%, soit environ trois foyers sur quatre, sont dans la trentaine et la quarantaine. Ils appartiennent à la génération active, qui joue un rôle central dans la vie de la collectivité locale, et si ces individus se sont réfugiés, on peut penser que c'est le fruit d'une décision murement réfléchie. Ainsi, 83,6\% des foyers avec des enfants mineurs ont pris la décision de partir, moins pour se protéger des dangers de la radioactivité que pour " protéger leur(s) enfant(s)».

- La profession des personnes : $82 \%$ des hommes travaillaient à plein temps, $12,7 \%$ étaient des travailleurs indépendants, exception faite de deux foyers où l'homme a donné sa démission. Quant aux formes de travail des femmes, du fait que c'est la génération en âge d'élever des enfants, $38,2 \%$ se sont déclarées femmes au foyer dites "sans emploi ", mais on compte aussi $32,7 \%$ de femmes qui travaillaient à plein temps. Cela s'explique par le fait que beaucoup de femmes sont devenues chefs de famille (quatre femmes ont divorcé avant de partir comme réfugiées,

données sur le refuge et le questionnaire. (N.D.T.) 
deux sont venues seules, une est mère célibataire, une autre est veuve), mais aussi que, plus généralement, beaucoup de femmes continuent de travailler après le mariage.

\section{2. Première étape : comportements après l'accident nucléaire}

Comment les plaignants se sont-ils comportés immédiatement après l'accident nucléaire?

La grande majorité des plaignants qui sont, rappelons-le, des réfugiés hors zone, a pris connaissance de l'accident nucléaire, par les médias (94,6\%), ou par «des amis ou des connaissances" (17,9\%). 52,7 \% ont dit " avoir ensuite vérifié à la TV ou sur les réseaux sociaux " ces informations, et $49 \%$ «avoir pris des mesures personnelles telles que fermer les fenêtres ». Ainsi, immédiatement après avoir entendu parler de l'accident nucléaire, nombreux sont les plaignants à avoir eu des comportements actifs, volontaires. Plus encore, $52,7 \%$ ont décidé d'évacuer, $32,7 \%$ sont "partis aussitôt avec toute la famille se réfugier quelque part ", $20 \%$ des mères sont "parties aussitôt avec les enfants". Le temps du séjour dans un refuge a été variable, 70,9\% d'entre eux ont vécu une vie de réfugiés allant de 3 semaines à 3 mois. Ici transparaissent le dynamisme et l'esprit d'initiative des plaignants de Kyoto, capables de chercher comment se procurer des informations sur les risques liés à la radioactivité et de décider par eux-mêmes. Partir comme réfugié entraîne beaucoup de sacrifices. Et c'est la menace ressentie devant les émissions radioactives qui les a poussés à vouloir s'échapper, à fuir, y compris des mères avec leurs enfants.

Malgré l'absence de consignes, $87,5 \%$ «ont cherché sur Internet » comment s'enfuir, notamment «inquiets pour la santé des enfants ou des foetus" (68,8\%). 56,3\% des plaignants disent "qu'ils n'avaient pas confiance dans les comptes rendus du gouvernement ", méfiance qui les a poussés à mesurer la radioactivité autour de chez eux $(61,8 \%)$ et à se dire que le risque était très élevé (60\%). La méfiance et l'inquiétude vis-à-vis des informations officielles étaient fortes : plus précisément, 77,1\% ont ressenti un danger à «ne pas pouvoir utiliser les comptes rendus du gouvernement et des collectivités locales » et $65,7 \%$ « sont devenus plus inquiets à l'écoute des comptes rendus de la commune». Notamment, en ce qui concerne les informations sur la dangerosité de la radioactivité, les plaignants ont consulté le Net mais aussi leur réseau d'amis vivant à l'étranger, 
les amenant à percevoir la différence entre les informations communiquées au Japon et ailleurs; et si certains ont mis leur confiance " dans les informations obtenues sur Internet » (76,5\%), ils ont par ailleurs éprouvé une forte méfiance pour " l'information officielle émanant du gouvernement " $(52,9 \%)$, «les réunions d'explication organisées par les municipalités et le gouvernement " $(29,4 \%)$. Ce que critique bon nombre de plaignants, c'est l'attitude du médecin Yamashita Jun.ichi 山下俊一 qui, après l'accident nucléaire de Tchernobyl, avait sonné l'alerte sur la dangerosité de la radioactivité, et qui, après l'accident, a complètement changé de position, faisant des conférences sur l'absence de danger de la radioactivité à Fukushima.

Beaucoup de plaignants ont affirmé avoir décidé alors de s'enfuir dans le Kansai après avoir vu le professeur de l'université de Tokyo, Kosako Toshiso 小佐古敏荘, conseiller du porte-parole du gouvernement, pleurer quand le gouvernement a décidé de relever jusqu'à $20 \mathrm{mSv}$ le seuil d'acceptation dans les cours des écoles primaires et secondaires et protester en disant qu' « il était inadmissible d'un point de vue scientifique d'appliquer une telle mesure aux enfants ".

\section{3. Cheminement jusqu'au refuge actuel}

Le choix du refuge actuel témoigne du même degré d'initiative et d'autonomie que pour les autres rubriques : $91 \%$ des plaignants déclarent qu' "ils n'avaient pas de consignes mais qu'ils ont beaucoup cherché " et dans la même proportion (71\%), qu' « inquiets pour leur santé future, ils sont devenus suspicieux vis-à-vis des comptes rendus du gouvernement ». Cette forte crainte s'expliquait par l'apparition de nombreux signes avantcoureurs de dysfonctionnements physiques observés au sein de la famille. Sont répertoriés des symptômes tels que "saignement de nez des enfants " $(41,2 \%)$, "rhume, fièvre " $(23,5 \%)$ et "diarrhées » (20,6 \%), y compris chez les parents, de façon répétée, et des symptômes autres (67,7 \%) tels que mycoplasme, otite, conjonctivite qui, avant l'accident n'étaient pas ressentis, mais sont devenus fréquents. Sur ces points, la comparaison montre des chiffres beaucoup plus élevés que dans le cas de Niigata où les pourcentages respectifs sont de 13,5 \%, 2,8 \%, et 2,2 \% (Takahashi \& Koike 2018 : 63), nous faisant comprendre qu'en observant les changements somatiques chez leurs proches, les plaignants de Kyoto ont directement été confrontés 
à la peur de la radioactivité, et poussés à partir. Ils ont pensé non pas aux départements limitrophes tels que Niigata ou Yamagata, mais à l'Ouest du Japon, où la pollution n'était pas à craindre.

Le choix du refuge actuel s'est accompagné de fortes inquiétudes; sont évoqués "l'inquiétude financière " $(92,7 \%)$, "le chagrin de quitter son emploi, de changer d'emploi » $(65,5 \%)$, c'est-à-dire la conscience de l'augmentation des dépenses liées à un déplacement lointain et l'inévitable question de l'emploi (changement ou démission). Ajoutées à cela, d'autres craintes telles que "la séparation familiale " $(49,1 \%)$, "le changement d'école pour les enfants " $(47,3 \%)$ montrent que la famille était la principale préoccupation. Tout particulièrement, parmi les réfugiés de Kyoto, le sentiment de la distance qui les séparait de la famille restée sur place, distance non seulement géographique mais aussi culturelle puisque se réfugier dans le Japon de l'Ouest était perçu comme se rendant dans une région, le Kansai, où les conditions naturelles, la façon de parler et les habitudes étaient différentes de ce qu'ils connaissaient. Ce refuge s'est donc accompagné du sentiment de perte du " pays natal " (furusato ふるさ そ) : un grand nombre de personnes ont évoqué «l'inquiétude d'être séparé de la famille avec laquelle on est habitué à vivre " $(80 \%)$, "la mauvaise conscience d'abandonner son pays natal " (69,1\%).

La raison principale de s'installer dans le Kansai au point d'éprouver un tel sentiment de perte était "le faible taux de radiation" (92,9\%). Une grande différence avec les plaignants de Niigata apparaît ici ; le choix qu'ont fait les réfugiés hors zone de se réfugier à Niigata est principalement motivé par le fait qu' " il y avait un système de location de logements provisoires " $(47,6 \%)$, suivi d'une raison géographique, à savoir " un accès direct par l'autoroute" $(40,2 \%)$ et enfin, en moindre proportion, "le bas niveau de radioactivité " (31,7\%) (Takahashi \& Koike 2018 : 66). Alors pourquoi choisir Kyoto? Les plaignants de Kyoto qui avaient donné la priorité avant tout à la fuite loin de la pollution radioactive ont recherché sur Internet et les médias un lieu " qui offrait un système d'accueil des réfugiés » $(62,5 \%)$, et ont décidé de s'y installer. La situation d'alors apparaît clairement dans les notes d'un plaignant qui a appris qu' « on louait des logements HLM aux réfugiés volontaires, non pas par les médias, mais en cherchant au hasard sur Internet, en recueillant des informations auprès d'individus qu'il n'avait jamais vus". 
Le département de Kyoto proposait des logements HLM habituellement destinés aux fonctionnaires, et la grande majorité des plaignants y a logé. (76,8\%). Mais les conditions vétustes de ces logements construits plus de cinquante ans auparavant, ont poussé une partie des plaignants $(25 \%)$ à déménager vers des " logements privés».

Y a-t-il eu accord ou non pour partir comme réfugiés? Si $66,1 \%$ ont décidé de partir "tous ensemble, avec toute la famille», montrant ainsi que dans l'ensemble le départ s'est fait d'un commun accord familial, en revanche, dans $14,3 \%$ des cas «l'accord entre membres de la famille a été difficile » et dans 7,1\% des cas " on s'est accordé dans la famille, mais l'accord avec la parentèle a été difficile». Les antagonismes et les ruptures après le départ avec la parentèle restée sur place n’ont pas été résolus, aboutissant dans 13,3\% des cas, à un divorce, ce qui a accentué les difficultés rencontrées par les mères et leurs enfants.

\section{4. La difficile vie de réfugiés des mères avec leurs enfants}

Que des mères avec leurs enfants aient décidé d'évacuer sans leur mari est un des phénomènes marquants créés par la catastrophe nucléaire de Fukushima $^{6}$. Dans $55,4 \%$ des cas du procès de Kyoto, la mère seule et les enfants sont devenus des réfugiés, le père restant sur place alors que dans $39,3 \%$ des cas, " toute la famille est partie ensemble sans séparation ", soit un rapport de 1,4. Ainsi les foyers à avoir choisi l'évacuation des femmes et des enfants sont plutôt nombreux.

À cause de ce choix de l'éloignement, les visites du père voyageant jusqu'au Kansai sont plus ou moins fréquentes - 40,7 \% une fois par mois dans $40,7 \%$ des cas, et $18,5 \%$ une fois tous les deux mois - soit presque $60 \%$. Pour ces pères qui ont choisi de rester sur place, la plus grande souffrance est " d'être séparés de leur femme et de leurs enfants " $(92,6 \%)$, et "l'augmentation de la charge économique " (92,6\%). Ces deux aspects sont complètement différents de ce qui est observé dans le cas de Niigata dont la proximité géographique permet aux pères de rendre visite à leur famille deux fois par mois et plus (94,8 \%) (Takahashi et al. 2018 : 68).

6. Il existe déjà de nombreux études et reportages sur la situation des mères réfugiées avec leurs enfants (Yamane 2013; Yoshida 2016; Toda et al. 2016). 
Ainsi les personnes qui se sont réfugiées dans le lointain Kansai pour fuir la pollution radioactive font l'expérience de la tristesse de la séparation familiale, d'une communication devenue difficile, les exposant au risque d'effondrement de la famille, voire de divorce.

L'éclatement du foyer en raison du fait du refuge de la mère et des enfants a-t-il pris fin? Lors de la rédaction des mémoires, si 52,9\% des personnes n'avaient pas retrouvé un foyer normal, 35,3\% des foyers avaient pu recomposer le foyer avec l'arrivée du père dans le lieu de refuge, et seulement $8,8 \%$ s'étaient retrouvés grâce au retour de la mère et des enfants.

Si $68,9 \%$ des foyers déclaraient ne pas voir de détérioration des relations entre époux du fait de la vie de réfugiés, 17,8\% disaient « que les querelles étaient nombreuses ", et 13,3\% (soit 6 foyers) pensaient qu'en menant des vies séparées, le risque de divorce était grand.

La proximité géographique de Niigata a créé une situation différente où $5,3 \%$ des foyers ont divorcé. En raison du manque de communication causé par la distance et de l'accroissement des divergences, le problème de l'isolement des mères vivant dans le Kansai ressort clairement.

\section{5. Effets de la vie de refugié sur les enfants}

La vie de refugié a grandement influencé les familles, et particulièrement les enfants. $73 \%$ des enfants ont changé d'école, perturbation à laquelle s'ajoute le dépaysement apporté par le déplacement dans le Kansai où la façon de parler et de penser est perçue comme étant différente. Les rubriques suivantes : " perte des amis » $(83,3 \%)$, « instabilité psychologique » $(56,7 \%)$, "absence d'amis dans la nouvelle école» (46,7\%), montrent des taux de réponse élevés, taux différents de ce qui ressort de l'enquête sur la vie de refugié à Niigata, plus proche géographiquement et culturellement où les taux de réponses respectivement de 38,5\%,29,5\% et 22,4\%, se révèlent être moitié moins élevés que ceux de Kyoto. Ces chiffres résonnent comme un cri de détresse des enfants réfugiés à Kyoto.

Le changement d'école a rendu les enfants physiquement vulnérables. 55,9 \% des enfants ont développé " des symptômes qui proviendraient des effets de la radioactivité ", et $17 \%$ des enfants présentent " des symptômes psychologiques». Plus encore, le développement conjoint de plusieurs symptômes parmi de nombreux enfants réclamerait une aide urgente. 
Si on considère l'influence générale de la vie de réfugié sur les enfants, le fait que seuls 9,8\% déclarent "ne pas avoir subi d'influence " montre bien l'ampleur des souffrances vécues par les enfants. On doit y ajouter les cas de brimades que subissent les enfants originaires de Fukushima à l'école, ce qui conduit $17,3 \%$ (un enfant sur six) d'entre eux à refuser d'aller à l'école ou à s'enfermer chez eux, situation qu'on peut qualifier d'anormale.

La souffrance des enfants, et celle de leurs mères qui les voient tous les jours apparaissent dans les enquêtes menées sur la base des mémoires.

\section{6. Conditions de vie et difficultés des réfugiés}

Tout comme les enfants, les adultes ont vu leur vie considérablement modifiée par l'expérience de réfugiés. La première des difficultés est le poids de la charge économique que 96,4\% des foyers (tous à l'exception de deux) soulignent. De même, nombreux sont les foyers qui mentionnent «les efforts pour affronter la vie » $(81,8 \%)$, « l'augmentation des dépenses de la vie quotidienne " $(76,3 \%)$, qui marquent la détérioration des conditions économiques suite au déplacement. Cette situation est due à « l'apparition d'une période de chômage ", qui concerne 54,4\% des hommes et 60,4\% des femmes. Plus encore, être réfugié a entraîné des pertes profondes dans la vie professionnelle. Outre la "perte de revenus " $(50 \%), 52,3 \%$ des hommes ressentent « la perte de l'envie de travailler », et $50 \%$ « la perte de leur plan de carrière ".

Les raisons de ces difficultés économiques sont claires. Étant réfugiés hors zone, 96,4\% des plaignants de Kyoto ont à affronter une injustice radicale concernant l'attribution des compensations financières. Par rapport aux réfugiés de la zone de retour difficile et de la zone d'habitation restreinte, qui ont reçu des dédommagements de 100000 yens par mois, jusqu'à la levée des restrictions, les réfugiés hors zone, même ceux vivant à l'intérieur du département de Fukushima, n’ont reçu, dans le cas des femmes enceintes et des enfants qu'une somme de 680000 yens, les autres adultes recevant des dédommagements de 80000 yens seulement ${ }^{7}$. Il est

7. Selon les calculs du professeur Yokemoto de l'université municipale d'Osaka, pour une famille de quatre personnes venant d'une zone de retour difficile, les compensations financières s'élèvent à 58000000 yens, à 28800000 yens si on est réfugié d'une zone 
clair que ces sommes ne peuvent compenser ni l'augmentation du poids des dépenses dues aux frais engagés pour se réfugier et pour continuer une double vie, ni la dépréciation de l'environnement économique qui naît de la vie de réfugié. En fait, ces "difficultés dues à la charge économique " sont également mentionnées dans les mémoires de Niigata, à 55,9\% pour les réfugiés de la zone d'évacuation, et 78,7 \% pour les réfugiés hors zone. Comme première difficulté rencontrée dès le début de la vie de réfugié, $39 \%$ des réfugiés de la zone d'évacuation et 76,4\% des réfugiés hors zone avaient souligné "l'augmentation des dépenses financières ", soit une différence considérable par rapport aux plaignants de Kyoto (Takahashi \& Koike 2018 : 67, 2019 : 92). Qu'ils soient réfugiés de la zone d'évacuation ou hors zone, les réfugiés de Niigata ou de Kyoto devraient ressentir de façon identique, l'augmentation de la charge économique produite par l'éloignement du lieu de vie qui avait été le leur jusqu'alors : aussi ressentir ou non une différence dans cette situation naît uniquement de la différence entre les sommes de compensations et de dédommagements versées par Tepco. Ce sont des dispositifs qu'on peut seulement qualifier d'absurdes.

Dans les mémoires, outre ces difficultés économiques, 67,2\% des plaignants évoquent "l'anxiété devant l'impossibilité d'envisager l'avenir ", et sur le plan des relations humaines, la détérioration de la santé psychique et physique. Nous reviendrons ultérieurement sur le rapport à l'avenir, mais d'abord considérons les difficultés rencontrées dans les relations humaines.

En ce qui concerne les relations familiales, 32,6 \% déclarent qu' " il n'y a pas eu de détérioration " dans les relations entre parents/enfants et frères/ sœurs, même si, par exemple, 11,6 \% disent qu'il y a eu " une détérioration temporaire " qu'il a été possible de surmonter. Toutefois, avec les beaux-parents, la situation a été plus délicate : $14 \%$ des gens ont souligné " une détérioration temporaire ", et 9,3\% « une vraie rupture ", manifestation d'une détérioradation des relations. Les relations avec les amis ont été davantage touchées : 32,6\% des gens ont déclaré "une détérioration temporaire des relations » et $27,9 \%$ " une rupture réelle ", ce qui montre

à habitation restreinte, à 19200000 yens si on est réfugié d'une zone de préparation à la levée de la restriction, et de 7200000 yens si on vient d'une zone de préparation au refuge en urgence alors que dans le cas d'une famille réfugiée hors zone, elle ne reçoit que 1680000 yens (Yokemoto 2015:39). 
à quel point la jalousie et la rupture surgissent facilement entre les parents ayant des enfants du même âge.

Quelles relations amicales se sont formées sur le lieu du refuge? $51 \%$ des plaignants éprouvent un fort sentiment de solitude, d'isolement, et 22,5\% l'expliquent par « les médisances à l'égard des réfugiés ». En revanche, plus nombreux sont les plaignants $(77,6 \%)$ qui expriment de la reconnaissance vis-à-vis de leur entourage, disant qu' « il leur est arrivé d'être secouru par des gens sympathiques ». Également, nombreux sont les plaignants qui évoquent le renforcement des liens horizontaux, par "l'entraide entre réfugiés ". C'est en raison d'une telle entraide que ces réfugiés, parfois même des mères et leurs enfants seuls, ont pu affronter la vie dans un lieu inconnu.

Si l'on regarde les conditions de santé, les hommes, évoquent le plus fréquemment "l'apparition de symptômes psychiques » (22,9\%), suivi dans 14,6 \% des cas de « l'apparition de symptômes qui pourraient être dûs aux effets de la radioactivité ». Dans le cas des femmes, 30,1\% évoquent ces mêmes symptômes, et dans un taux assez élevé aussi "l'apparition de symptômes psychiques » $(21,8 \%)$. Un simple calcul montre que plus de la moitié des femmes souffrent d'une maladie, chiffres qui reflètent la lourdeur et la dureté de la vie de réfugiées.

En ce qui concerne la souffrance psychique, le premier problème chez les hommes est «la souffrance, le danger qui accompagne les déplacements " (60,4\% des cas), qui traduit le poids ressenti à rendre visite à leurs femmes et leurs enfants dans le lieu de refuge.

De plus, bon nombre des hommes se plaignent du changement ou de la perte survenue sur le plan professionnel : 52,1\% évoquent la souffrance qui accompagne la "perte du goût au travail », $52 \%$ "la contrainte à la démission ou au changement professionnel » et 47,9\% « la diminution du salaire ". Pour les femmes, comme pour les hommes, la première souffrance est "la souffrance, le danger qui accompagne le déplacement» (89\%), suivi à $61,8 \%$ "des désordres physiques et psychiques provoqués par le stress ", montrant que les efforts psychiques et physiques pèsent sur leur état. Une autre particularité est la mention par les femmes de deux peines celle d' " avoir été contraintes de séparer leur mari des enfants » $(52,7 \%)$, et celle d' " avoir fait changer d'école les enfants » (49,1\%). Ainsi, alors que les hommes accordent de l'importance à leur travail, les femmes à qui revient la protection de la famille, sont plus concernées par la charge psychique d'assurer le rôle de parent. 


\section{7. A propos du retour}

Seuls 4 foyers, soit $4 \%$, sont retournés vivre dans leur lieu d'origine avant la rédaction des mémoires du procès, invoquant comme raison à ce retour "la charge économique », ce qui montre les difficultés économiques provoquées par la double vie des mères réfugiées. $96 \%$ des foyers ne sont pas retournés dans leur lieu de vie d'origine, en raison de «la radioactivité et le danger pour la santé ", montrant bien que la pollution radioactive est considérée comme un problème nullement réglé. L'État et les collectivités locales insistent sur la diminution de la radioactivité grâce au travail de décontamination, mais ainsi que l'explicite l'un des plaignants, «la décontamination n'est pas terminée, et la pollution du sol ne diminue toujours pas ». Certains sont en colère contre Tepco et l'État qui "n'ont pas réglé l'accident $"$.

À propos du seuil de radioactivité qui rendrait le retour envisageable, $72,1 \%$ avancent "le niveau d'avant l'accident", et 11,6\% qu'ils n'ont " rien de concret en tête, mais que c'est encore élevé ", soit un total de $85 \%$. Comme ces niveaux ne sont pas encore atteints, les plaignants pensent ne pas pouvoir retourner chez eux pour le moment.

\section{8. Réclamation d'indemnisations et appréciation}

Plus de la moitié des plaignants ont réclamé des indemnisations, directement à Tepco dans 61,1\% des cas, via le processus Alternative Dispute Resolution $^{8}$ dans $50 \%$ des cas. Mais un fort mécontentement s'exprime devant le montant des indemnisations reçues : $93 \%$ se déclarent « complètement insatisfaits ».

De façon générale, les réponses à l'accident nucléaire apportées par Tepco, l'État, le département de Fukushima et les municipalités d'origine des plaignants au procès de Kyoto ont mécontenté les individus, respectivement à hauteur de 96,4\%, 96,4\%, 63,8 \% et 47,8\%. La différence avec ce qui a été vu au procès de Niigata est nette : le taux de mécontentement est plus faible, avec tout particulièrement une grande appréciation

8. ADR est un système alternatif de versements de compensations financières placé sous la direction du ministère de l'Éducation (Monbukagakushō 文部科学省, MEXT). (N.D.T.) 
des réponses apportées par le département de Fukushima et les municipalités. Ainsi, pour les réfugiés hors zone, le fort mécontentement concernait $94,1 \%, 88,1 \%, 20,1 \%$ et $21,2 \%$, alors que pour les réfugiés de la zone, les taux respectifs étaient de $96,1 \%, 84,3 \%, 5,9 \%$ et $7,7 \%$.

Les plaignants du procès de Kyoto avaient un point de vue plus critique : la question de la souffrance et de la faible fréquence des déplacements dans le Kansai par les pères, les dépenses à engager pour organiser le retour dans la région d'origine sont des raisons pour lesquelles le mécontentement est grand parmi les plaignants de Kyoto.

\section{Considérations sur la situation actuelle}

En résumé, que pouvons-nous dire des caractéristiques et des comportements des réfugiés tels que les montrent les mémoires du procès de Kyoto? Ces réfugiés, tout comme ceux apparaissant dans les autres procès, appartiennent principalement à la génération qui élève encore de jeunes enfants et travaille. Ce qui est frappant ce sont leurs comportements déterminés et actifs, preuves de l'esprit d'autonomie de ces plaignants. On a vu la façon dont ils ont collecté des informations, les ont examinées et après avoir eu connaissance du risque de pollution radioactive qu'ils commençaient d'observer sur la santé de leurs proches, ils ont pris la décision de fuir au loin, dans le Kansai pour protéger leurs enfants de cette pollution.

Toutefois, ces familles ont été confrontées à des difficultés plus grandes qu'elles ne l'avaient prévu. Ces difficultés étaient avant tout d'ordre économique, et certaines personnes ont été contraintes au chômage ou au changement d'emploi, auxquelles s'est ajoutées la souffrance ressentie du fait de devoir travailler dans un environnement inhabituel au contact de personnes nouvelles, et de la diminution des revenus. Plus encore, il est arrivé que, afin de maintenir le niveau de revenu, le mari soit resté vivre dans le lieu de vie d'avant la catastrophe et que seuls la femme et les enfants soient partis, provoquant séparation familiale et isolement, manque de communication, et une double vie entraînant un accroissement des dépenses. Il en a résulté que 13,3\% des foyers ont été poussés au divorce.

Les difficultés vécues par les plaignants ne se sont pas limitées à la dimension économique. 
Tous, parents comme enfants, ont perdu les liens avec leurs amis et leur parentèle, ont été séparés de l'environnement naturel auquel ils étaient habitués, et vivent dans un monde nouveau où les différences culturelles sont considérées comme des facteurs renforçant le sentiment de solitude et d'isolement. Dans cette situation marquée par la rareté des relations humaines, la difficulté à s'adapter à l'environnement nouveau, la confrontation à des paroles malintentionnées voire à la discrimination, ce sont les enfants qui ont été les plus affectés et $1 / 6$ ont été amenés à refuser d'aller à l'école ou à devenir des retirants (bikikomori 引きこもり). Confrontées directement à ces souffrances, 61,8\% des mères ont commencé à souffrir de "déséquilibres physique et psychologique provoqués par le stress ».

Difficultés économiques, perte de relations sociales, augmentation du stress psychologique, perte des relations avec le pays natal, la liste des difficultés et des souffrances est longue, mais ne s'arrête pas là. Les plaignants de Kyoto éprouvent des souffrances et des difficultés invisibles et insaisissables par les chiffres. Dans l'environnement quotidien qui est le leur, les individus échangent avec la nature, nouent des relations avec des amis et des enseignants, des supérieurs hiérarchiques, et ainsi se développent en apprenant et en travaillant. Vivre s'inscrit dans le temps : chacun forme son identité sur la base d'un passé et en préparant l'avenir; dans ce processus peuvent être construites des relations humaines stables et une famille. Voilà ce qu'est la vie en société et ce qui doit être considéré comme un droit de toute la population. Mais l'accident nucléaire de Fukushima en a complètement privé les plaignants du procès de Kyoto. Il a tranché le lien que les plaignants avaient avec leur passé, leur a fait perdre la protection de la société, et les a privés de leur avenir puisqu'ils ne savent pas jusqu'à quand ils devront vivre cette vie de réfugiés; ainsi l'identité de chacun des plaignants devient incertaine et leur vie présente instable. Les chiffres montrent bien cela : 91,1 \% d'entre eux déclaraient éprouver «l'angoisse de l'avenir ", et trois ans après la rédaction des mémoires du procès, 67,2 \% d'entre eux évoquaient encore "l'angoisse de ne pas pouvoir envisager l'avenir ». Les deux tiers des plaignants ont le sentiment d'avancer dans le noir, sans pouvoir envisager l'avenir, de ne pas recevoir suffisamment de garantie, et de devoir vivre, exposés à divers dangers.

Dans le présent article, nous avons cherché à comprendre les difficultés que les plaignants affrontaient, les souffrances dans lesquelles ils étaient et avec lesquelles ils devaient vivre. S’il n’y avait pas eu l'accident nucléaire, 
ces difficultés et ces souffrances n'existeraient pas. En outre, il n'y a aucune raison que les plaignants assument la moindre responsabilité vis-à-vis de ces difficultés et de ces souffrances, ni qu'ils aient la moindre obligation de les accepter. Malgré cela, leur vie a complètement été bouleversée par cet accident, les privant même de futur. Aussi, le questionnement du présent article sur la façon dont les plaignants se sont vus imposés cette douloureuse situation nous amène à penser qu'il serait erroné de croire que sa suppression permettrait aux réfugiés de retrouver une vie paisible et stable. Parler de difficultés et de souffrances imposées aux plaignants n'est pas approprié; il serait plus exact de dire que l'accident nucléaire les a privés du droit de vivre à l'abri de telles difficultés et souffrances, du droit de constuire une vie meilleure en entretenant des relations avec la nature et les gens en société.

Le philosophe italien Giorgio Agamben cite les mots d'Aristote " on est né pour vivre mais on existe pour mener une vie essentiellement bonne ${ }^{9}$ ». Dans la Grèce ancienne, le fait de vivre tout simplement et le fait de vivre en recherchant une vie bonne en société sont clairement distingués. Alors que vivre une vie bonne est appelé « bios " et est la forme de vie recherchée en société, vivre tout simplement est appelé «Zoe » qui signifie vivre, activité commune à tous les êtres vivants, et Agamben nomme cela une "vie nue » la vie exclue de la protection de la société et des règles (Agamben $2003: 7-20)$.

Le droit de «vivre une vie bonne » est un droit attribué à toute la population, que la Constitution japonaise garantit (article 13) comme suit : "Tous les citoyens devront être respectés comme individus. Leur droit à la vie, à la liberté, à la poursuite du bonheur, dans la mesure où il ne fait pas obstacle au bien-être public, demeure le souci suprême du législateur et des autres responsables du gouvernement". Selon ce texte, en 2012, l'année qui a suivi le désastre, le Parlement a ratifié à l'unanimité la loi pour l'aide aux victimes de l'accident nucléaire. La loi s'intitule « loi relative à la promotion de mesures concernant les aides à la vie quotidienne des victimes pour protéger et soutenir la vie quotidienne des habitants à commencer

9. Dans Les Politiques, Aristote écrit que : «Et la communauté achevée formée de plusieurs villages est une cité dès lors qu'elle a atteint le niveau de l'autarcie pour ainsi dire complète; s'étant donc constituée pour permettre de vivre, elle permet une fois qu'elle existe une vie heureuse. " (Aristote 1993 : 91) 
par les enfants suite à l'accident nucléaire de la centrale nucléaire Tepco » (denryoku genshiryoku jiko ni yori hisai shita kodomo o hajime to suru jümin nado no seikatsu o mamori sasaeru tame no hisaisha no seikatsu shien nado ni kansuru shisaku no suishin ni kansuru hōritsu 電力原子力事故により被災 した子どもをはじめとする住民等の生活を守り支えるための被災者の生活支援 等に関する施策の推進に関する法律, dite 《loi d'aide aux victimes dont les enfants " [kodomo nado hisaisha shien hō 子ども等被災者支援法]). Dans l'article 2 énonçant que « chaque victime peut, selon sa propre volonté, choisir soit de revenir dans la région d'avant le déplacement, soit de résider dans une région qui fait l'objet d'une aide conformément à l'article 8 alinéa 1 , quel que soit le choix fait par les victimes, une aide appropriée doit être attribuée ", il est clairement précisé que toutes les victimes ont le droit de décider librement d'évacuer leur lieu de vie et que l'État et les communes doivent leur fournir une aide appropriée.

Malgré les textes de la Constitution et de la loi, les réfugiés qui ont fui la pollution radioactive de l'accident nucléaire sont contraints de mener une "vie nue " sans aucune protection. Aussi, ne devrions-nous pas poser la question suivante? Le droit à " une vie bonne » dont ont été privés les réfugiés hors zone par la Société d'électricité de Tokyo, qui est l'entreprise responsable, n'est-il convertissable qu'en compensations financières - pour les enfants et les femmes enceintes : 400000 yens (dans le cas de refuge : 600000 yens), et pour les adultes 80000 yens? Les mesures actuelles prises par l'État et les collectivités locales qui abandonnent ces individus à la «vie nue » sont absolument inacceptables. Qu'est-ce qui pourrait les légitimer?

Traduit du japonais par Anne GONON. 


\section{Bibliographie}

AgAmben Giorgio アガンベン・ジョルジョ 2003

Homo sakeru : shuken kenryoku to hakidashino sei ホモ・サケル一主権権力 と剥ぎ出しの生 (Homo sacer : le pouvoir souverain et la vie nue), TAKAKUWA Kazumi 高桑和巳 (trad.), Tokyo, Ibunsha 以文社.

\section{Aristote アリストテレス 2018}

Seijigaku 政治学 (Les Politiques), UChIYAmA Katsutoshi 内山勝利, KANZAKI Shigeru 神崎繁, NAKAHATA Masashi 中畑正志, AizAWA Yasutaka 相澤康隆, SEGUCHI Masahisa 瀬口 昌久 (trad.), Arisutoteresu zenshū 17 アリストテレス全集17, Iwanami shoten 岩波書店.

\section{ENDō Noriko 遠藤典子 2013}

Genshiryoku songai baishō seido no kenkyū 原子力損害賠償制度の研究 (Études sur le système d'indemnisations des dommages nucléaires), Tokyo, Iwanami shoten.

Kwansei gakuin daigaku saigai fukkō seido kenkyūjo 関西学院大学災害復 興制度研究所 (Centre de recherches sur le système de reconstruction des désastres de l'université Kwansei gakuin) 2015

Genpatsu hinan hakusho 原発避難白書 (Le livre blanc des réfugiés du nucléaire), Jinbun shoin 人文書院.

SATō Yoshiyuki 佐藤嘉幸 \& $T_{A G U C H I}$ Takumi 田口卓臣 2016 Datsu genpatus no tetsugaku 脱原発
の哲学 (Philosophie de la sortie du nucléaire), Jinbun shoin.

TAINAKA Masato 田井中雅人 \& TsuJıмото Eimi ツジモト•エイミ 2018

Hyōryū suru tomodachi : Amerika no hibaku saiban 漂流するトモダチ:アメリ カの被ばく裁判 (Procès des irradiés aux États-Unis : soldats abandonnés), Asahi shinbun shuppan 朝日新聞出版.

TAKAHASH Wakana 高橋若菜 \& KoIKE Yuka 小池由香 2018

«Genpatsu hinan seikatsushi (1) jiko kara hon hinan ni itaru michi : genpatsu hinansha Niigata sosho/genkoku 237 setai no chinjutsusho o moto to shita ryōteki kōsatsu » 原発避難生活史(1) 事 故から本避難に至る道一原発避難者新潟 訴訟・原告237世帯の陳述書をもととし た量的考察 (Histoires de vie en refuge [1] De l'accident au refuge actuel : considerations quantitatives basées sur les plaidoyers de 237 foyers plaignants au procès des réfugiés du nucléaire de Niigata), Utsunomiya daigaku kokusaigakubu kenkyū ronshū 宇都宮大学 国際学部研究論集, $46: 51-71$.

TAKAHASH Wakana \& KoIKE Yuka 2019 « Genpatsu hinan seikatsushi (2) jiko kara hon hinan ni itaru michi : genpatsu hinansha Niigata sosho : genkoku 237 setai no chinjutsusho o moto to shita ryōteki kōsatsu » 原発避難生活 史(2) 事故から本避難に至る道一原発避 難者新潟訴訟・原告237世帯の陳述書 をもととした量的考察 (Histoires de vie en refuge [2] De l'accident au refuge actuel : considerations quantitatives basée sur les plaidoyers de 237 foyers 
plaignants au procès des réfugiés du nucléaire de Niigata), Utsunomiya daigaku kokusaigakubu kenkyū ronshū, 47: 91-111.

TsuJIUCHI Takuya 辻内环也 \& MASUDA Kazutaka 増田和高 (dir.) 2019

Fukushima no iryō jinruigaku : genpatsu jiko.shien no firudowāku フクシマの医療 人類学一原発事故・支援のフィールドワーク (Anthropologie médicale de Fulushima : enquête de terrain sur l'accident de la centrale nucléaire et l'aide), Tokyo, Tomi shobō遠見書房.

\section{TODA Noriki 戸田典樹 (dir.) 2016}

Fukushima genpatsu jiko hyōryū suru jishu hinanshatachi : jittai chōsa kara mita kadai to shakaiteki shien no arikata 福島 原発事故: 漂流する自主避難者たち一実態調 査から見た課題と社会的支援のあり方 (L'accident de la centrale nucléaire de Fukushima : la dérive des réfugiés volontaires. Questions et modes d'aides vus à partir de l'enquête des conditions réelles), Tokyo, Akashi shoten 明石書店.

\section{Matsutani Michiru 松谷満, Woncheol} Sung 成元哲, UshiJima Kayo 牛島佳代 \& SAKAGUCHI Yusuke 坂口祐介 2014 «Fukushima genpastu jikogo ni okeru "jishu hinan" no shakaiteki kiteiin : Fukushima-ken Nakadōri chiiki no boshi chōsa kara» 福島原発事故後における“自主 避難”の社会的規定因一福島県 中通り地域の母子調査から (Causes sociales déterminantes de l'évacuation volontaire après l'accident nucléaire de Fukushima : sur une enquête mèreenfant dans la région de Nakadōri du département de Fukushima), Ajia taiheiyō rebyūアジア太平洋レビュー， université d'économie et de droit d'Osaka, $11: 68-77$.

\section{SeIYAma Kazuo 盛山和夫 2004}

Shakai chōsahō nyūmon 社会調査法入門 (Introduction à la méthode de l'enquête sociale), Yūhikaku bukkusu 有斐閣ブック ス, Tokyo, Yūhikaku 有斐閣.

\section{Sung Woncheol (dir.) 2015}

Owaranai hisai no jikan : genpatsu jiko ga fukushimaken nakadōri no oyako ni ataeru eikyō 終わらない被災の時間一原発 事故が福島県中通りの親子に与える影響 (Un désastre sans fin : influence de l'accident nucléaire sur les enfants du centre du département de Fukushima), Fukuoka, Sekifūsha 石風社.

\section{YAmAne Sumika 山根純佳 2013}

"Genpatsu jiko ni yoru "boshi hinan" mondai to sono shien : Yamagata-ken ni okeru hinansha chōsa no dēta kara » 原発事故による“母子避難”問題とその支援一 山形県における避難者調査のデータから (La question des « mères et enfants réfugiés » à cause de l'accident nucléaire et leur aide : données de l'enquête sur les réfugiés menée dans le département de Yamagata), Yamagata daigaku jinbungakubu kenkyū nenpō 山形大学人 文学部研究年報, $10: 37-51$.

\section{Үокемото Masafumi 除本理史 2013}

Genpatsu baishōo tou : aimaina sekinin, honrō sareru hinansha 原発賠償を問う一 曖昧な責任、翻弄される避難者

(Questionner les indemnisations du nucléaire : des responsabilités vagues, des réfugiés malmenés), Iwanami 
bukkuretto 岩波ブックレット, Iwanami shoten.

Yокемото Masafumi 2016

«Fukushima genpatsu jiko ni yoru

hinansha e no kasetsu jūtaku no kyōyo

shūryō nitsuite » 福島原発事故による避難

者への仮設住宅の供与終了について (De la

fin de l'offre des logements provisoires

aux réfugiés de l'accident de la centrale nucléaire), Keiei kenkyū 経営研究, 68 (3) :

35-51.

Yoshida Chia 吉田千亜 2016

Rupo boshi hinan : kesareyuku genpatsu

jiko higaisha ルポ母子避難一消されゆ

<原発事故被害者 (Reportage sur les

mères et enfants réfugiés : la disparition

des victimes de l'accident nucléaire),

Iwanami shinsho 岩波新書.

WatANABE Akihiro 渡辺明宏 2016

«Gentei sareru jishu hinansha no songai baishō » 限定される避難者の損害賠償 (Des indemnisations limitées pour les réfugiés volontaires), in TODA Noriki 戸田典樹 (dir.), Fukushima genpatsu jiko hyōryū suru jishu hinanshatachi : jittai chōsa kara mita kadai to shakaiteki shien no arikata, Tokyo, Akashi shoten : 156-168. 